\title{
Co-activação dos músculos flexores e extensores da articulação do joelho em condições isocinéticas
}

\author{
Rui S. Gonçalves ${ }^{1}$ \\ J. Páscoa Pinheiro ${ }^{2}$
}

https://doi.org/10.5628/rpcd.05.02.215

\author{
${ }^{1}$ Escola Superior de Tecnologia da Saúde de Coimbra \\ Portugal \\ ${ }^{2}$ Universidade de Coimbra \\ Faculdade de Medicina e \\ Faculdade de Ciências do Desporto e Educação Física \\ Portugal
}

\begin{abstract}
Co-activation of the knee joint flexors and extensors muscles in isokinetics conditions

The co-activation of the knee flexors and extensors muscles has been largely examined resorting to surface electromyography. The aim of this review is to present findings related to the contribution of the antagonist electromyographic (EMG) activity to the motor's actions of the knee joint complex in isokinetics conditions. The factors angular position, type of muscle action and angular velocity influence the antagonist EMG activity levels. In isokinetics conditions the antagonist activation contributes to the restriction of the resultant joint moment and the maintenance of the joint stability.
\end{abstract}

A co-activação dos músculos flexores e extensores do sistema articular do joelho tem sido amplamente examinada com recurso à electromiografia de superfície. Esta revisão tem com objectivo apresentar achados relativos à contribuição da actividade electromiográfica (EMG) antagonista para a acção motora do complexo do joelho, em condições isocinéticas. Os factores posição angular, tipo de acção muscular e velocidade angular influenciam os níveis de actividade EMG antagonista. Em condições isocinéticas, a activação antagonista contribui para a restrição do momento articular resultante e para a manutenção da estabilidade articular.

Palavras-chave: co-activação, joelho, electromiografia de superfície, dinamometria isocinética.
Key Words: co-activation, knee, surface electromyography, isokinetic dynamometry. 


\section{INTRODUÇÃO}

A prática de actividades desportivas expressa-se, fundamentalmente, através de tarefas e acções motoras dinâmicas extremamente exigentes sob o ponto de vista biocinético, uma vez que demandam forças musculares e articulares consideráveis. Enquanto movem as articulações, os músculos são os principais responsáveis pela colocação de cargas mecânicas sobre as superfícies articulares, interferindo na integridade e estabilidade articular (1). Na prática desportiva, o joelho é sujeito a múltiplas solicitações mecânicas, inerentes ao desempenho de habilidades e actividades específicas das modalidades praticadas, revelando-se uma articulação intrinsecamente instável $(2,3)$. Devido às suas características anatómicas e funcionais, esta articulação é submetida a forças e momentos resultantes particularmente elevados, sobretudo durante os movimentos de extensão e de flexão, dependendo da interacção músculo-ligamentar para assegurar a estabilidade funcional (4-12). A ocorrência de actividade da musculatura antagonista durante a contracção voluntária da musculatura agonista, mecanismo designado de co-activação muscular (13), parece estar associada a acções de estabilização e protecção articular $(8,9,14,35)$. A co-activação dos músculos flexores e extensores da articulação do joelho tem sido amplamente investigada em condições isocinéticas, com recurso à electromiografia de superfície (16). A dinamometria isocinética, embora não reproduza as condições reais da prática desportiva devido à especificidade do tipo de movimento que autoriza (i.e., implica a manutenção de velocidade constante), permite analisar a influência de vários factores (ex.: posição angular, tipo de acção muscular, velocidade angular) na co-activação muscular (16). Diversos trabalhos demonstraram a presença de actividade EMG antagonista em exercícios isocinéticos de extensão e flexão do joelho $(8,9,14$, 38). Por exemplo, Kellis \& Baltzopoulos (30) relataram valores de actividade EMG antagonista isquiotibial, normalizada como percentagem da actividade EMG agonista do mesmo músculo recolhida nas mesmas condições de velocidade angular, posição angular e tipo de acção muscular, de $24 \%$ (extensão concêntrica - $30^{\circ}$.s. ${ }^{-1}$ ), $15 \%$ (extensão excêntrica $30^{\circ}$.s. ${ }^{-1}$ ), $28 \%$ (extensão concêntrica - $150^{\circ}$. s. $^{-1}$ ) e $20 \%$ (extensão excêntrica $-150^{\circ}$.s. $^{-1}$ ), para uma amostra de 12 alunas universitárias de ciências do desporto e educação física (idade: $20,5 \pm 2,9$ anos; peso: $64,0 \pm 6,1 \mathrm{~kg}$; estatura: 1,67 $\pm 0,02 \mathrm{~m}$ ). Esta revisão tem com objectivo apresentar achados relativos à contribuição da actividade electromiográfica (EMG) antagonista para a acção motora do complexo do joelho, em condições isocinéticas.

\section{INFLUÊNCIA DA POSIÇÃO ANGULAR, TIPO DE ACÇÃO MUSCULAR E VELOCIDADE ANGULAR NA ACTIVAÇÃO ANTAGONISTA.}

A utilização sincronizada de sistemas de electromiografia de superfície e dinamometria isocinética permite quantificar, em simultâneo, a actividade EMG dos músculos agonistas e antagonistas registada no decurso de movimentos isocinéticos de extensão e flexão do joelho. Estes movimentos analíticos podem ser executados através da totalidade da amplitude de movimento, na sequência de acções musculares concêntricas e excêntricas, e utilizando um largo espectro de velocidades $(39,43)$. Por este motivo, a dinamometria isocinética permite manipular convenientemente os factores posição angular, tipo de acção muscular e velocidade angular. Kellis (16) disponibiliza um artigo de revisão no qual discute com rigor os métodos que têm sido utilizados na determinação da actividade antagonista dos músculos quadriceps e isquiotibiais, nomeadamente em condições isocinéticas.

Estudos recentes examinaram a influência da posição angular $(14,17,19,26,28,29)$, tipo de acção muscular $(9,17,19,30)$ e velocidade angular $(20$, $22,23,26,30,35,38)$ na activação muscular antagonista, durante esforços isocinéticos de extensão e flexão do joelho. A informação adquirida através destas investigações é útil para ajudar a compreender a contribuição da activação antagonista para o momento articular resultante e para a estabilidade articular. A activação antagonista é mais elevada nas partes iniciais e finais da amplitude de movimento $(14,17$, $19,26,28,29)$. Em toda a amplitude articular, a actividade EMG antagonista relaciona-se inversamente com o braço do momento do músculo, ou seja, com a distância perpendicular entre a linha de acção do músculo e o centro instantâneo de rotação da articulação $(26,28)$. Baratta e col. (28) recolheram simultaneamente a actividade EMG dos múscu- 
los isquiotibiais e quadriceps durante movimentos isocinéticos concêntricos de extensão e flexão do joelho, executados a baixa velocidade $\left(15^{\circ}\right.$. s. $\left.^{-1}\right)$. Foi utilizada uma amostra de vinte e quatro indivíduos, 17 atletas (idade média: 22 anos) e 7 não atletas (idade média: 27 anos). A representação gráfica da actividade EMG antagonista (normalizada como percentagem da actividade EMG agonista do mesmo músculo, recolhida em iguais condições de velocidade angular e posição angular) versus posição articular apresentou, grosseiramente, a forma de U. Baratta e col. (28) propõem que este padrão de actividade antagonista pretende compensar a variação do braço do momento do músculo, na tentativa de manter a produção de momento antagonista constante. $\mathrm{Na}$ fase final do sector angular, a actividade antagonista aumentada parece contribuir também para a desaceleração do segmento, assegurando a integridade da articulação $(26,28,29,36)$.

A activação antagonista regista valores significativamente mais elevados durante acções concêntricas, comparativamente a acções excêntricas, dos músculos agonistas $(9,17,19,30)$. Kellis \& Baltzopoulos (30), num estudo já referido, obtiveram valores percentuais de activação antagonista isquiotibial que ilustram claramente este padrão, independentemente da velocidade angular: $24 \%$ (extensão concêntrica) versus $15 \%$ (extensão excêntrica), a $30^{\circ} .{ }^{\circ} .^{-1} ; 28 \%$ (extensão concêntrica) versus $20 \%$ (extensão excêntrica), a $150^{\circ}$.s. ${ }^{-1}$. Paralelamente, o momento articular resultante é superior durante acções musculares excêntricas, enquanto que a activação agonista é mais elevada durante acções musculares concêntricas $(18,27,30,43,45)$. Com isto em mente, é sugerida a presença de um mecanismo neural inibitório que previne o aumento excessivo do momento articular resultante (assegurando a integridade do sistema músculo-esquelético) e para o qual contribui a activação muscular antagonista $(18,30)$.

O valor médio de actividade EMG antagonista, registado para a totalidade da amplitude de movimento durante a extensão e a flexão do joelho, aumenta com o incremento da velocidade angular $(20,22,23$, $26,30,35,38)$, embora a actividade EMG antagonista registada na fase inicial desses mesmos movimentos diminua ligeiramente (26). Hagood et al. (26) estudaram o efeito da velocidade angular na activa- ção antagonista dos músculos isquiotibiais e quadriceps em 6 adultos do sexo masculino (idade mínima: 20 anos; idade máxima: 43 anos). Foi demonstrado o acréscimo dos níveis de actividade antagonista, aumentando a velocidade de $15^{\circ}$.s. ${ }^{-1}$ até $240^{\circ}$. s. $^{-1}$, dos músculos isquiotibiais (128\%) e do quadriceps (110\%), nos últimos $40^{\circ}$ de amplitude dos movimentos de extensão e de flexão, respectivamente. $\mathrm{Na}$ fase inicial desses movimentos, o incremento da velocidade angular resultou numa diminuição ligeira da actividade antagonista dos músculos isquiotibiais (24\%) e do quadriceps (8\%). Segundo Hagood et al. (26), estas alterações na activação antagonista devem-se ao aumento das fases de aceleração e desaceleração nos movimentos realizados a velocidades mais elevadas. Deste modo, a ligeira diminuição da activação antagonista na fase inicial ajuda a aceleração do segmento, e o aumento da activação antagonista na amplitude final facilita a travagem dinâmica do segmento, prevenindo o dano dos ligamentos e cápsula articular. Importa aqui referir que, embora do ponto de vista teórico a principal característica do movimento isocinético seja a manutenção de velocidade constante (aceleração zero), na prática há aceleração e desaceleração em todos os movimentos realizados num dinamómetro isocinético. Isto, porque independentemente da velocidade a que se desenrolam os movimentos, estes iniciam e terminam com velocidade zero (46). As propriedades viscoelásticas do músculo também podem contribuir para explicar o efeito da velocidade angular na actividade antagonista (35). Osternig et al. (36) sugerem que durante o movimento concêntrico de extensão do joelho, realizado a velocidades elevadas, a força dos músculos do quadriceps excede a tensão viscoelástica dos músculos isquiotibiais, exigindo activação antagonista para controlo e desaceleração da perna.

\section{CONTRIBUIÇÃO DA ACTIVAÇÃO ANTAGONISTA PARA O MOMENTO ARTICULAR RESULTANTE}

A performance muscular tem sido avaliada através da metodologia isocinética com ênfase na acção muscular agonista. No entanto, reconhecendo a presença da co-activação muscular durante a execução do movimento isocinético, parece razoável assumir que a medida revelada pelo dinamómetro equivale simplesmente ao momento da força líquido gerado na 
articulação, ou seja, ao momento articular resultante (16). O momento articular resultante representa a soma de todos os momentos exercidos ao redor da articulação (16). Uma vez que os músculos extensores e flexores da articulação do joelho produzem momentos de força em sentidos opostos, em termos analíticos o momento articular resultante corresponde à subtracção do momento agonista pelo momento antagonista $(14,16,17)$. Consequentemente, o momento articular resultante é mais baixo na presença de co-activação antagonista, comparativamente a uma situação de silêncio antagonista (47).

Vários estudos demonstraram a presença de actividade EMG antagonista do grupo muscular isquiotibial aquando da contracção concêntrica máxima do grupo muscular quadriceps, no decorrer do movimento de extensão do joelho $(9,14,15,17,18,24,26$, $28,29,36)$. Deste modo, a determinação do momento antagonista é essencial para entender a contribuição da activação antagonista para o momento articular resultante (17). Métodos baseados em modelos matemáticos, integrando o processamento de dados electromiográficos, têm sido utilizados para estimar os momentos articulares agonista e antagonista durante o movimento isocinético de extensão do joelho $(8,14,17,26,28)$. Por exemplo, Kellis \& Baltzopoulos (17) e Aagaard et al. (14), utilizando métodos muito similares, estimaram o momento antagonista isquiotibial a partir de relações EMGmomento determinadas durante contracções agonistas do mesmo tipo (grupo isquiotibial: contracção excêntrica; grupo quadriceps: contracção concêntrica). Kellis \& Baltzopoulos (17) recolheram o momento articular resultante durante os movimentos concêntricos e excêntricos de extensão e flexão do joelho, realizados a $30^{\circ}$.s. ${ }^{-1}$, numa amostra de 10 homens (idade: $23 \pm 1,5$ anos; peso: $74 \pm 3,8 \mathrm{~kg}$; estatura: $1,74 \pm 0,04 \mathrm{~m})$. Paralelamente recolheram a actividade EMG dos músculos vasto medial e biceps femoral. Para determinar o momento antagonista isquiotibial foi calculada a relação integral electromiográfico (iEMG)/momento em diferentes níveis de esforço muscular. Em seguida, as curvas iEMG/momento foram ajustadas utilizando polinómios de segundo grau. Finalmente, os polinómios foram utilizados para estimar o momento antagonista exercido pelos isquiotibiais a partir do iEMG antagonista: 42,92
N.m durante acções concêntricas e 28,97 N.m durante acções excêntricas, dos músculos agonistas. Aagaard et al. (14) recolheram o momento articular resultante e os sinais EMG dos músculos vasto medial, vasto lateral, recto femoral e biceps femoral durante movimentos concêntricos e excêntricos de extensão do joelho, realizados a $30^{\circ}$. . $^{-1}{ }^{-1}$, numa amostra de 16 indivíduos do sexo masculino (idade: 23,5 $\pm 3,4$ anos; peso: $73,2 \pm 5,8 \mathrm{~kg}$; estatura: $179 \pm 4$ $\mathrm{cm})$. A actividade EMG antagonista isquiotibial recolhida durante a extensão concêntrica (contracção máxima dos quadriceps) foi convertida em momento antagonista com base nas relações EMG/momento determinadas durante a extensão excêntrica (contracção máxima dos isquiotibiais), e vice versa. Foram utilizadas equações matemáticas para construir as relações EMG/momento para os músculos dos grupos quadriceps e isquiotibial, respectivamente, uma vez que a activação antagonista esteve presente em ambos os movimentos de extensão. Foi estimado um momento isquiotibial antagonista de aproximadamente 30 N.m.

Os resultados apresentados pelos estudos anteriores indicam que o momento articular gerado pela activação antagonista isquiotibial interfere significativamente no momento articular resultante, durante esforços isocinéticos de intensidade máxima, realizados pelos músculos do quadriceps $(14,17)$. Estudos anteriores haviam apresentado resultados similares, ainda que o momento antagonista estimado representasse uma menor contribuição para o momento articular resultante $(8,26,28)$. No entanto, esta discrepância parece resultar em grande parte da utilização de diferentes metodologias na normalização do sinal EMG antagonista (48). Nos estudos mais antigos, o efeito do tipo de acção muscular não foi considerado $(8,26,28)$. Este facto, por si só, parece contribuir para justificar a apresentação de valores de momento antagonista mais baixos, uma vez que a determinado nível de actividade EMG corresponde maior momento durante contracções excêntricas, comparativamente a contracções concêntricas (17). O momento antagonista isquiotibial é quase constante em toda a amplitude articular $(8,9,14,17,26$, 28). Isto, porque a actividade antagonista aumenta nos sectores inicial e final do movimento, enquanto que o momento articular exercido pelo músculo, 
enquanto agonista, é mais baixo nessas mesmas amplitudes. Assim, um valor de actividade antagonista durante as fases inicial e final do movimento corresponde a um momento articular mais baixo, enquanto que o mesmo valor de actividade antagonista na amplitude média corresponde a um valor de momento mais elevado. Aagaard et al. (14), num estudo já referido, estimaram níveis quase constantes de momento antagonista isquiotibial (30 N.m) em toda a amplitude articular. No entanto, a sua contribuição para o momento articular resultante aumenta à medida que o movimento se aproxima da extensão completa $(14,17)$. No estudo de Aagaard et al. (14), o momento antagonista estimado correspondeu a $30-74 \%$ do momento articular resultante, na amplitude articular próxima da extensão completa $\left(10-30^{\circ}\right)$. Neste extremo da amplitude articular do joelho, os músculos extensores apresentam menor vantagem mecânica, enquanto que os flexores exercem o momento articular máximo (17).

O momento antagonista é significativamente mais elevado durante acções concêntricas, comparativamente a acções excêntricas, dos músculos agonistas (quadriceps) $(9,17)$. Kellis \& Baltzopoulos (17), num estudo já referido, estimaram valores máximos de momento antagonista isquiotibial correspondentes a 42,92 N.m durante acções concêntricas e a 28,97 N.m durante acções excêntricas, dos músculos agonistas. Durante as acções musculares excêntricas, a rácio de momento articular resultante por unidade de actividade electromiográfica agonista é superior à registado em acções concêntricas $(27,30,43,45)$. Durante as acções concêntricas dos músculos agonistas (quadriceps), os músculos antagonistas (isquiotibiais) trabalham excentricamente, produzindo maior quantidade de momento articular antagonista, quando comparado com acções concêntricas dos antagonistas (isquiotibiais), durante esforços excêntricos do agonista (quadriceps) $(9,17)$. Com isto em mente, Kellis \& Baltzopoulos $(17,30)$ sugerem que os valores mais elevados de actividade antagonista registados durante as acções concêntricas dos agonistas podem explicar parcialmente a obtenção de valores mais baixos de momento articular resultante nessas condições, comparativamente a acções excêntricas dos agonistas.

\section{CONTRIBUIÇÃO DA ACTIVAÇÃO ANTAGONISTA PARA A ESTABILIDADE ARTICULAR}

Ainda que os ligamentos sejam considerados os responsáveis primários pela estabilidade estática de uma articulação, os estudos da activação antagonista exibida durante a contracção voluntária máxima do grupo muscular quadriceps têm conferido crescente protagonismo ao papel regulador do grupo muscular isquiotibial na manutenção da estabilidade articular $(8,28,29,36,49,50)$, uniformização da pressão entre as superfícies articulares e regulação da impedância mecânica da articulação do joelho (28). Vários estudos evidenciaram que, durante o movimento isocinético de extensão do joelho, os músculos do quadriceps geram forças de deslize anterior e de rotação interna da tíbia, especialmente no sector angular mais próximo da extensão completa $(6,8$, $11,51,54)$. O momento articular gerado pela activação antagonista dos músculos isquiotibiais parece contribuir para contrabalançar as forças de deslize anterior $(9,14,17,28,29,49)$. Para além disso, a activação antagonista do músculo biceps femoral, em particular, parece representar um mecanismo de oposição à rotação interna, uma vez que a localização lateral da sua inserção confere capacidade para provocar rotação externa $(14,34)$. Para corroborar esta sugestão, importa referir que a actividade EMG antagonista apresentada pelo músculo semitendinoso, cuja localização lateral da respectiva inserção poderia reforçar a rotação interna induzida pelos músculos do quadriceps, é cerca de três vezes inferior à apresentada pelo músculo biceps femoral $(14,34)$. Conforme Basmajian \& De Luca (47), os movimentos balísticos executados a velocidades elevadas, presentes em condições reais da prática desportiva, apresentam um padrão trifásico de activação dos músculos agonistas e antagonistas: fase inicial revela elevada activação agonista com silêncio antagonista, possibilitando a aceleração do segmento; fase intermédia - caracterizada por redução da activação agonista e incremento da activação antagonista; fase final - denota elevada activação agonista e antagonista, permitindo a desaceleração do segmento e a cessação do movimento. Nestas circunstâncias, o papel da co-activação muscular parece estar mais relacionado com a aceleração/desaceleração do 
segmento do que com a estabilização da articulação. Durante o exercício isocinético, o padrão de activação antagonista não reproduz o padrão trifásico característico dos movimentos balísticos executados a grande velocidade, isto é, a activação antagonista é mais elevada nas partes iniciais e finais da amplitude de movimento $(14,17,19,26,28,29)$. Nestas condições, a velocidade angular é controlada externamente pelo dinamómetro e aos músculos agonistas é exigido o desenvolvimento constante de força máxima ao longo de todo o sector angular. Deste modo, na amplitude média e final do movimento, os músculos antagonistas têm que assumir de forma exclusiva as funções de aceleração/desaceleração, sem qualquer assistência dos músculos agonistas (26). Pelo facto da velocidade do movimento isocinético ser controlada exteriormente, a co-activação muscular parece actuar essencialmente ao nível da estabilização da articulação do joelho, assumindo apenas um papel secundário na promoção de condições favoráveis às funções de aceleração/desaceleração da perna (26).

A activação antagonista dos músculos isquiotibiais, importante na regulação da estabilidade da articulação do joelho, parece ser mediada através de mecanismos centrais e/ou periféricos $(14,28,29)$. A existência de um arco reflexo desencadeado pelo aumento da tensão exercida sobre os mecanoreceptores localizados no ligamento cruzado anterior, tendo como resposta a activação dos músculos isquiotibiais, foi demonstrada em preparações animais $(29)$ e em humanos $(55,56)$. A sobrecarga do ligamento cruzado anterior, criada pela contracção do grupo muscular quadriceps, é reduzida pela activação antagonista, especialmente na fase final do movimento de extensão $(8,9)$. Quando existe lesão do ligamento cruzado anterior, um outro arco reflexo, provavelmente mediado por mecanoreceptores proprioceptivos (nos músculos isquiotibiais e/ou na cápsula articular), parece desencadear a resposta antagonista (29). Alguns autores sugerem que a alteração do padrão normal de co-activação dos músculos isquiotibiais pode resultar de disfunção do ligamento cruzado anterior $(20,57)$. Solomonow et al. (29) demonstraram a capacidade dos músculos isquiotibiais para corrigirem a subluxação anterior da tíbia, em indivíduos com lesão do ligamento cruzado anterior, durante a contracção voluntária máxima do grupo muscular quadriceps. Outros autores observaram o aumento da co-activação dos isquiotibiais em indivíduos com lesão do ligamento cruzado anterior $(25,58,59)$.

Devido ao comportamento da actividade antagonista isquiotibial em função da posição angular, ou seja, relaciona-se inversamente com o braço do momento do músculo, Baratta et al. (28) propõem os receptores cinestésicos da cápsula articular, através de uma complexa rede polisináptica, como a principal origem aferente determinante do padrão de activação antagonista.

Aagaard et al. (14) sugerem que a extensão voluntária do joelho, realizada com intensidade máxima, representa uma circunstância na qual o momento articular antagonista gerado pelos músculos isquiotibiais pode ser antecipado, através de mecanismos de mediação central, para regular o stress tensional induzido sobre o ligamento cruzado anterior pela contracção do grupo muscular quadriceps. Este argumento parece estar de acordo com a hipótese de condução neural comum (ou activação neural proporcional), segundo a qual o sistema nervoso central controla de forma sistemática e coordenada um grupo ou grupos de unidades motoras, não só de músculos agonistas como também de músculos antagonistas (60). Isto porque o mecanismo de activação neural proporcional agonista-antagonista parece estar presente em alguns movimentos, especialmente quando existe incerteza sobre a tarefa motora requerida, ou durante a antecipação de resposta neuromuscular compensatória (60).

\section{CONCLUSÃO}

Um vasto número de estudos demonstraram a presença de actividade EMG antagonista durante os movimentos isocinéticos de extensão e de flexão da articulação do joelho. Foi evidenciado que os factores posição angular, tipo de acção muscular e velocidade angular (facilmente manipuláveis por dinamo- 
metria isocinética) influenciam os níveis de actividade EMG antagonista. No movimento concêntrico de extensão do joelho, o momento antagonista (estimado com base na actividade EMG antagonista isquiotibial) é quase constante ao longo de toda a amplitude de movimento e contribui significativamente para o momento articular resultante. $\mathrm{O}$ momento articular gerado pela activação antagonista dos músculos isquiotibiais parece contrabalançar as forças de deslize anterior e de rotação interna da tíbia, induzidas pelos músculos do quadriceps, contribuindo para a manutenção da estabilidade articular do joelho.

\section{CORRESPONDÊNCIA}

Rui Soles Gonçalves

Curso de Fisioterapia

Escola Superior de Tecnologia da Saúde de Coimbra Rua 5 de Outubro, S. Martinho do Bispo

Apartado 7006

3040-162 Coimbra

Portugal

ruigoncalves@estescoimbra.pt 


\section{REFERÊNCIAS BIBLIOGRÁFICAS}

1. Herzog W, Longino D, Clark A (2003). The role of muscles in joint adaptation and degeneration. Langenbecks Arch Surg 388(5):305-15.

2. Fadale PD, Hulstyn MJ (1997). Common athletic knee injuries. Clin Sports Med 16(3):479-99.

3. Cailliet R (1983). Knee pain and disability. 2 nd ed. Philadelphia: FA Davis.

4. Amarantini D, Martin L (in press). A method to combine numerical optimization and EMG data for the estimation of joint moments under dynamic conditions. Journal of Biomechanics, Corrected Proof.

5. Baltzopoulos V (1995). Muscular and tibiofemoral joint forces during isokinetic concentric knee extension. Clin Biomech (Bristol, Avon) 10(4):208-214.

6. Chow JW (1999). Knee joint forces during isokinetic knee extensions: a case study. Clin Biomech (Bristol, Avon) 14(5):329-38.

7. Lloyd DG, Besier TF (2003). An EMG-driven musculoskeletal model to estimate muscle forces and knee joint moments in vivo. J Biomech 36(6):765-76.

8. Kaufman KR, An KN, Litchy WJ, Morrey BF, Chao EY (1991). Dynamic joint forces during knee isokinetic exercise. Am J Sports Med 19(3):305-16.

9. Kellis E, Baltzopoulos V (1999). The effects of the antagonist muscle force on intersegmental loading during isokinetic efforts of the knee extensors. J Biomech 32(1):19-25

10. Kellis E (2001). Tibiofemoral joint forces during maximal isokinetic eccentric and concentric efforts of the knee flexors. Clin Biomech (Bristol, Avon) 16(3):229-36.

11. Nisell R, Ericson MO, Nemeth G, Ekholm J (1989). Tibiofemoral joint forces during isokinetic knee extension. Am J Sports Med 17(1):49-54.

12. Wei SH (2000). Dynamic joint and muscle forces during knee isokinetic exercise. Proc Natl Sci Counc Repub China B 24(4):161-8.

13. Psek JA, Cafarelli E (1993). Behavior of coactive muscles during fatigue. J Appl Physiol 74(1):170-5.

14. Aagaard P, Simonsen EB, Andersen JL, Magnusson SP, Bojsen-Moller F, Dyhre-Poulsen P (2000). Antagonist muscle coactivation during isokinetic knee extension. Scand $J$ Med Sci Sports 10(2):58-67.

15. Kellis E, Unnithan VB (1999). Co-activation of vastus lateralis and biceps femoris muscles in pubertal children and adults. Eur J Appl Physiol Occup Physiol 79(6):504-11.

16. Kellis E (1998). Quantification of quadriceps and hamstring antagonist activity. Sports Med 25(1):37-62.

17. Kellis E, Baltzopoulos V (1997). The effects of antagonist moment on the resultant knee joint moment during isokinetic testing of the knee extensors. Eur J Appl Physiol Occup Physiol 76(3):253-9.

18. Amiridis IG, Martin A, Morlon B, Martin L, Cometti G, Pousson M, et al (1996). Co-activation and tension-regulating phenomena during isokinetic knee extension in sedentary and highly skilled humans. Eur J Appl Physiol Occup Physiol 73(1-2):149-56.

19. Kellis E, Baltzopoulos V (1996). The effects of normalization method on antagonist activity during concentric and eccentric isokinetic knee extension and flexion. $J$ Electromyogr Kinesiol 6(4):235-45

20. Osternig LR, Caster BL, James CR (1995). Contralateral hamstring (biceps femoris) coactivation patterns and ante- rior cruciate ligament dysfunction. Med Sci Sports Exerc 27(6):805-8.

21. Oberg B, Moller M, Gillquist J, Ekstrand J (1986). Isokinetic torque levels for knee extensors and knee flexors in soccer players. Int J Sports Med 7(1):50-3.

22. Snow CJ, Cooper J, Quanbury AO, Anderson JE (1993). Antagonist cocontraction of knee flexors during constant velocity muscle shortening and lengthening. J Electromyogr Kinesiol 3(2):78-86

23. Snow CJ, Cooper J, Quanbury AO, Anderson JE (1995) Antagonist cocontraction of extensors during constant velocity muscle shortening and lengthening. J Electromyogr Kinesiol 5(3):185-92.

24. Bobbert MF, Harlaar J (1993). Evaluation of moment-angle curves in isokinetic knee extension. Med Sci Sports Exerc 25(2):251-9.

25. Grabiner MD, Weiker GG (1993). Anterior cruciate ligament injury and hamstring coactivation. Clin Biomech (Bristol, Avon) 8(4):215-9.

26. Hagood S, Solomonow M, Baratta R, Zhou BH D'Ambrosia R (1990). The effect of joint velocity on the contribution of the antagonist musculature to knee stiffness and laxity. Am J Sports Med 18(2):182-7.

27. Tesch PA, Dudley GA, Duvoisin MR, Hather BM, Harris RT (1990). Force and EMG signal patterns during repeated bouts of concentric or eccentric muscle actions. Acta Physiol Scand 138(3):263-71.

28. Baratta R, Solomonow M, Zhou BH, Letson D, Chuinard R, D'Ambrosia R (1988). Muscular coactivation. The role of the antagonist musculature in maintaining knee stability. Am J Sports Med 16(2):113-22.

29. Solomonow M, Baratta R, Zhou BH, Shoji H, Bose W, Beck $\mathrm{C}$, et al (1987). The synergistic action of the anterior cruciate ligament and thigh muscles in maintaining joint stability. Am J Sports Med 15(3):207-13.

30. Kellis E, Baltzopoulos V (1998). Muscle activation differences between eccentric and concentric isokinetic exercise. Med Sci Sports Exerc 30(11):1616-23

31. Kellis E (1999). The effects of fatigue on the resultant joint moment, agonist and antagonist electromyographic activity at different angles during dynamic knee extension efforts. J Electromyogr Kinesiol 9(3):191-9.

32. Kellis E, Kellis S (2001). Effects of agonist and antagonist muscle fatigue on muscle coactivation around the knee in pubertal boys. J Electromyogr Kinesiol 11(5):307-18.

33. Kellis E (2003). Antagonist moment of force during maximal knee extension in pubertal boys: effects of quadriceps fatigue. Eur J Appl Physiol 89(3-4):271-80.

34. Miller JP, Croce RV, Hutchins R (2000). Reciprocal coactivation patterns of the medial and lateral quadriceps and hamstrings during slow, medium and high speed isokinetic movements. J Electromyogr Kinesiol 10(4):233-9.

35. Weir JP, Keefe DA, Eaton JF, Augustine RT, Tobin DM (1998). Effect of fatigue on hamstring coactivation during isokinetic knee extensions. Eur J Appl Physiol Occup Physiol 78(6):555-9.

36. Osternig LR, Hamill J, Lander JE, Robertson R (1986). Coactivation of sprinter and distance runner muscles in isokinetic exercise. Med Sci Sports Exerc 18(4):431-5.

37. Croce RV, Miller JP (2003). The effect of movement velocity and movement pattern on the reciprocal co-activation of the hamstrings. Electromyogr Clin Neurophysiol 43(8):451-8. 
38. Miller JP, Croce RV (2002). Effect of movement velocity and movement pattern on the root mean square and the median frequency of the electromyographic activity of the quadriceps during isokinetic testing. Isokinetics and Exercise Science 10(4):193-8.

39. Baltzopoulos V, Brodie DA (1989). Isokinetic dynamometry. Applications and limitations. Sports Med 8(2):101-16.

40. Cabri J (1991). Isokinetic strength aspects of human joints and muscles. Crit Rev Biomed Eng 19(2-3):231-59.

41. Delitto A (1990). Isokinetic dynamometry. Muscle Nerve 13 Suppl:S53-7.

42. Kannus P (1994). Isokinetic evaluation of muscular performance: implications for muscle testing and rehabilitation. Int J Sports Med 15 Suppl 1:S11-8.

43. Kellis E, Baltzopoulos V (1995). Isokinetic eccentric exercise. Sports Med 19(3):202-22.

44. Aagaard P, Simonsen EB, Andersen JL, Magnusson SP, Halkjaer-Kristensen J, Dyhre-Poulsen P (2000). Neural inhibition during maximal eccentric and concentric quadriceps contraction: effects of resistance training. J Appl Physiol 89(6):2249-57.

45. Westing SH, Cresswell AG, Thorstensson A (1991). Muscle activation during maximal voluntary eccentric and concentric knee extension. Eur J Appl Physiol Occup Physiol 62(2):104-8.

46. Brown LE (2000). Isokinetics in human performance. Champaign: Human Kinetics.

47. Basmajian JV, De Luca CJ (1985). Muscles alive, their functions revealed by electromyography. 5 th ed. Baltimore: Williams \& Wilkins.

48. Gonçalves RS, Pinheiro JP (2003). Normalização da actividade electromiográfica antagonista recolhida no decurso de provas isocinéticas de extensão e de flexão da articulação do joelho. Ludens 17(2):47-50.

49. Renstrom P, Arms SW, Stanwyck TS, Johnson RJ, Pope MH (1986). Strain within the anterior cruciate ligament during hamstring and quadriceps activity. Am J Sports Med 14(1):83-7.

50. Yanagawa T, Shelburne K, Serpas F, Pandy M (2002). Effect of hamstrings muscle action on stability of the ACL-deficient knee in isokinetic extension exercise. Clin Biomech (Bristol, Avon) 17(9-10):705-12.

51. Beynnon BD, Fleming BC (1998). Anterior cruciate ligament strain in-vivo: a review of previous work. J Biomech 31(6):519-25.

52. Beynnon BD, Fleming BC, Johnson RJ, Nichols CE, Renstrom PA, Pope MH (1995). Anterior cruciate ligament strain behavior during rehabilitation exercises in vivo. $\mathrm{Am}$ J Sports Med 23(1):24-34.

53. More RC, Karras BT, Neiman R, Fritschy D, Woo SL, Daniel DM (1993). Hamstrings-an anterior cruciate ligament protagonist. An in vitro study. Am J Sports Med 21(2):231-7.

54. Hirokawa S, Solomonow M, Lu Y, Lou ZP, D'Ambrosia R (1992). Anterior-posterior and rotational displacement of the tibia elicited by quadriceps contraction. Am J Sports Med 20(3):299-306.

55. Tsuda E, Okamura Y, Otsuka H, Komatsu T, Tokuya S (2001). Direct evidence of the anterior cruciate ligamenthamstring reflex arc in humans. Am J Sports Med 29(1):83-7.

56. Dyhre-Poulsen P, Krogsgaard MR (2000). Muscular reflexes elicited by electrical stimulation of the anterior crucia- te ligament in humans. J Appl Physiol 89(6):2191-5.

57. Chu D, LeBlanc R, D'Ambrosia P, D'Ambrosia R, Baratta RV, Solomonow M (2003). Neuromuscular disorder in response to anterior cruciate ligament creep. Clin Biomech (Bristol, Avon) 18(3):222-30.

58. Williams GN, Barrance PJ, Snyder-Mackler L, Axe MJ, Buchanan TS (2003). Specificity of muscle action after anterior cruciate ligament injury. J Orthop Res 21(6):1131-7.

59. Doorenbosch CA, Harlaar J (2003). A clinically applicable EMG-force model to quantify active stabilization of the knee after a lesion of the anterior cruciate ligament. Clin Biomech (Bristol, Avon) 18(2):142-9.

60. De Luca CJ, Mambrito B (1987). Voluntary control of motor units in human antagonist muscles: coactivation and reciprocal activation. J Neurophysiol 58(3):525-42. 